\title{
Quantification of preeclampsia-related microRNAs in maternal serum
}

\author{
QIAN LI ${ }^{1}$, ANXIONG LONG ${ }^{1}$, LIANSHENG JIANG ${ }^{1}$, LEIMING CAI $^{1}$, \\ $\mathrm{LI} \mathrm{XIE}^{1}, \mathrm{JI}^{\prime} \mathrm{AN} \mathrm{GU}^{1}, \mathrm{XIONG} \mathrm{CHEN}^{2}$ and LONGYITAN ${ }^{1}$ \\ ${ }^{1}$ Department of Clinical Laboratory, Shanghai First People's Hospital Baoshan Branch; ${ }^{2}$ Department of Obstetrics \\ and Gynecology, Shanghai First People's Hospital Baoshan Branch, Shanghai 200940, P.R. China
}

Received May 22, 2015; Accepted July 15, 2015

DOI: $10.3892 /$ br.2015.524

\begin{abstract}
To identify the specific serum preeclampsia (PE)-related biomarkers, 10 microRNAs (miRNAs) were selected based on their reported aberrant (4 upregulated and 6 downregulated) expression in PE placenta. A total of 1,035 pregnant patients were enrolled. Finally, 32 pregnancies with PE and 32 healthy pregnancies were incorporated in the study. The expression of these 10 miRNAs in the different trimesters was determined by SYBR-Green reverse transcription-quantitative polymerase chain reaction. Compared with that in the healthy controls, the expression levels of miR-152, miR-183 and miR-210 in PE serum were higher in the second and third trimester, whereas the expression of miR-182 was only higher in the third trimester. The expression levels of 6 miRNAs (miR-1, miR-328, miR-363, miR-377, miR-500 and miR-584) that were downregulated in PE placenta showed no significant differences between pregnancies complicated by $\mathrm{PE}$ and healthy pregnancies throughout the 3 trimesters. Areas under the receiver operating characteristic [standard error (SE)] during the 20-24th gestational week for predicting PE were miR-152: 0.94 (SE, 0.026), miR-183: 0.97 (SE, 0.031) and miR-210: 0.93 (SE, 0.018). In conclusion, the expression levels of serum miR-152, miR-183 and miR-210 were elevated since the second trimester in pregnancies complicated with $\mathrm{PE}$, indicating their potentials as serum biomarkers for forecasting PE.
\end{abstract}

\section{Introduction}

Preeclampsia (PE) is a multi-systemic disorder characterized by the new onset of hypertension and proteinuria after the

Correspondence to: Dr Tan Longyi, Department of Clinical Laboratory, Shanghai First People's Hospital Baoshan Branch, 216 Mudanjiang Road, Shanghai 200940, P.R. China

E-mail: sybsfytly@163.com

Key words: preeclampsia, microRNA, serum, biomarker, reverse transcription-quantitative polymerase chain reaction 20th gestation week. PE affects $2-8 \%$ pregnancies worldwide and remains one of the leading causes of maternal and neonatal mortality and morbidity (1). Although the exact pathological mechanism has not been identified, the defective placenta is recognized to have a vital role in PE (2).

MicroRNAs (miRs or miRNAs) are endogenously expressed, small (19-25 nucleotides) non-coding RNAs that regulate the expression of different genes via post-transcriptional repression. Pineles et al (3) reported differential miR expression in PE placenta for the first time. Thereafter, more investigations were performed with regards to placental miRs in PE and numerous differential placental miRs were identified, including miR-210, miR-152 and miR-584 (4-6). These studies suggested a potential role of miRs in PE pathophysiology (7).

The differential placental miRs may be present in the circulation through possible secretory machinery from cells (8). Therefore, those miRs dysregulated in placenta may be detected in serum and serve as potential biomarkers for early screening or diagnosis of PE. The aim of the present study was to quantify 10 previously reported differential placental miRs in serum and evaluate their potential value in PE early screening or diagnosis. These $10 \mathrm{miRs}$ were chosen based on previous studies $(3,4,6,9,10$; Table I), which demonstrated differential miRs expression in placenta, including 4 upregulated miRs (miR-152, miR-182, miR-183 and miR-210) and 6 dowregulated miRs (miR-1, miR-328, miR-363, miR-377, miR-500 and miR-584).

\section{Materials and methods}

Serum collection. The study was approved by the Local Ethics Review Board of Shanghai First People's Hospital Baoshan Branch (Shanghai, China). Peripheral blood samples were obtained from 1,035 Chinese pregnancies who received routine obstetric examination in the hospital between January 2011 and February 2014. Informed consent was obtained from every subject prior to blood collection. According to the clinical data, 32 singleton pregnancies who delivered in the hospital complicated with PE were identified in the serum bank, and 32 healthy pregnant women without complications were selected as the control based on similar maternal age at delivery within a 1-year-old gap and the same gestational 
Table I. Characteristics of the 10 selected microRNAs.

\begin{tabular}{lll}
\hline microRNA & \multicolumn{1}{c}{ Sequence of microRNA } & \multicolumn{1}{c}{ Selection reason } \\
\hline miR-1 & 5'-UGGAAUGUAAAGAAGUAUGUAU-3' & Downregulated in PE placenta \\
miR-152 & 5'-UCAGUGCAUGACAGAACUUGG-3' & Upregulated in PE placenta \\
miR-182 & 5'-UUUGGCAAUGGUAGAACUCACACU-3' & Upregulated in PE placenta \\
miR-183 & 5'-UAUGGCACUGGUAGAAUUCACU-3' & Upregulated in PE placenta \\
miR-210 & 5'-CUGUGCGUGUGACAGCGGCUGA-3' & Upregulated in PE placenta \\
miR-328 & 5'-CUGGCCCUCUCUGCCCUUCCGU-3' & Downregulated in PE placenta \\
miR-363 & 5'-AAUUGCACGGUAUCCAUCUGUA-3' & Downregulated in PE placenta \\
miR-377 & 5'-AUCACACAAAGGCAACUUUUGU-3' & Downregulated in PE placenta \\
miR-500 & 5'-UAAUCCUUGCUACCUGGGUGAGA-3' & Downregulated in PE placenta \\
miR-584 & 5'-UUAUGGUUUGCCUGGGACUGAG-3' & Downregulated in PE placenta
\end{tabular}

PE, preeclamsia.

Table II. Clinical characteristics of the subjects.

\begin{tabular}{lcc}
\hline Characteristics & PE $(\mathrm{n}=32)$ & Control $(\mathrm{n}=32)$ \\
\hline Maternal age, year & $28.7 \pm 3.6$ & $28.1 \pm 3.8$ \\
Basic BMI, $\mathrm{kg} / \mathrm{m}^{2}$ & $22.7 \pm 3.1$ & $21.6 \pm 2.6$ \\
PE diagnosis week & $35.3 \pm 2.5$ & $\mathrm{NA}$ \\
Birth weight, $\mathrm{g}$ & $3,054.4 \pm 547.5$ & $3,366.9 \pm 437.6$ \\
\hline
\end{tabular}

PE, preeclampsia; BMI, body mass index; NA, not applicable.

week of blood sampling. The relevant clinical details for the pregnancies are shown in Table II. PE was defined as systolic pressure $>140 \mathrm{mmHg}$ or diastolic blood pressure $>90 \mathrm{mmHg}$ in 2 different occasions with proteinuria $(>0.3 \mathrm{~g} / 24 \mathrm{~h})$ after the 20th gestation week. All the serum were collected from different trimester pregnancies after $\geq 8 \mathrm{~h}$ fasting and maintained at $-80^{\circ} \mathrm{C}$ until used.

RNA extraction. Total RNA was extracted from $200 \mu \mathrm{l}$ serum using the miRNeasy Serum/Plasma kit (Qiagen, Hilden, Germany) following the manufacturer's instructions. Synthetic spike-in control Cel-miR-39-1 (Qiagen) was used to monitor miRNA purification and amplification. The RNAs were dissolved in $14 \mu \mathrm{l}$ RNase-free water.

miRNA reverse transcription-quantitative polymerase chain reaction $(R T-q P C R)$ analysis. miR RT-qPCR was performed as previously described. Briefly, total RNA ( $4 \mu \mathrm{l})$ was reverse transcribed using the miScript IIRT kit (Qiagen) in a $20 \mu \mathrm{l}$ total volume reaction system containing the miScript Primer Assay (Qiagen) on a PCR system (Bioer Technology Co., Ltd., Hangzhou, China) with the following thermal parameters: $60 \mathrm{~min}$ at $37^{\circ} \mathrm{C}$ and $5 \mathrm{~min}$ at $95^{\circ} \mathrm{C}$. Subsequently, RT-qPCR was performed on the 7500 Real-Time PCR System (Applied Biosystems, Foster City, CA, USA) using the miScript SYBR-Green PCR kit (Qiagen) in a total reaction volume of $20 \mu \mathrm{l}$ containing $2 \mu \mathrm{l}$ cDNA (200X diluted). The PCR thermal parameters were as follows: A total of $15 \mathrm{~min}$ at $95^{\circ} \mathrm{C}$,
40 cycles of $15 \mathrm{sec}$ at $94^{\circ} \mathrm{C}$ followed by $30 \mathrm{sec}$ at $55^{\circ} \mathrm{C}$, and $34 \mathrm{sec}$ at $70^{\circ} \mathrm{C}$. All the PCR reactions were run in triplicate. The expression levels of miRs were determined utilizing the comparative $\mathrm{C}_{\mathrm{T}}$ method relative to cel-miR-39-1 (11).

Statistical analysis. Expression levels of serum miRs were compared using the Mann-Whitney U test. Receiver operating characteristic curves were plotted and the area under curve (AUC) was estimated to illustrate the potential of miRNAs being a predictive biomarker. $\mathrm{P}<0.01$ was considered to indicate a statistically significant difference. All the statistical analysis was performed with SPSS 17.0 software (SPSS Inc., Chicago, IL, USA).

\section{Results}

Serum expression of the miRs ( $m i R-152$, miR-182, miR-183 and miR-210) elevated in PE placenta. Compared with those in the control group, the relative quantification of 3 miRs (miR-152, miR183 and miR-210) were elevated in pregnancies with PE since the 20th gestation week and maintained at a high level until the third trimester. The expression of miR-182 showed no difference between pregnancies with PE and healthy pregnancies in the first and second trimester. In the third trimester, the miR-182 level in pregnancies with PE was higher than that in the healthy pregnancies (Fig. 1).

Serum expression of the miRs (miR-1, miR-328, miR-363, miR-377, miR-500 and miR-584) that were reduced in $P E$ placenta. Throughout the 3 trimesters there were no significant differences of serum miRs (miR-1, miR-328, miR-363, miR-377, miR-500 and miR-584) that were reduced in PE placenta identified between pregnancies with PE and healthy pregnancies (Fig. 2).

Elevated miRNAs (miR-152, miR-183 and miR-210) during the 20-24th gestational weeks may predict later onset of preeclampsia. As is shown in Fig. 3, AUC during the 20-24th gestational weeks for predicting PE of miR-152, miR-183 and miR-210 were 0.94 (SE, 0.026), 0.97 (SE, 0.031) and 0.93 (SE, 0.018), respectively. 
A

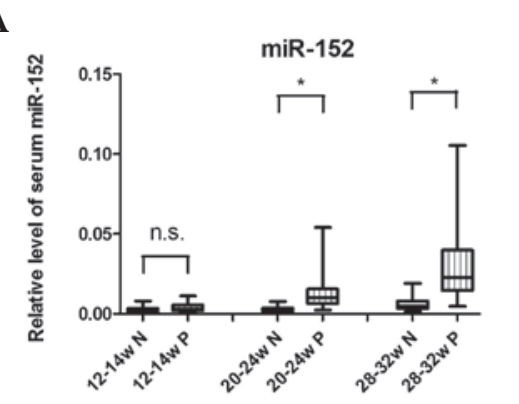

$\mathbf{C}$

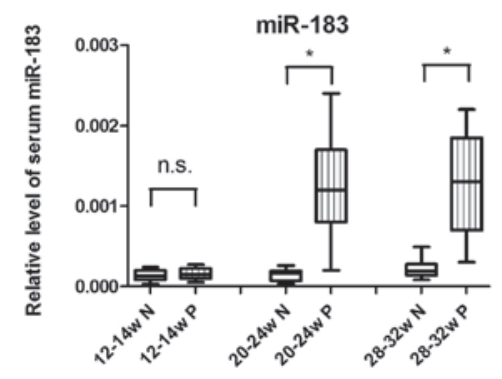

B

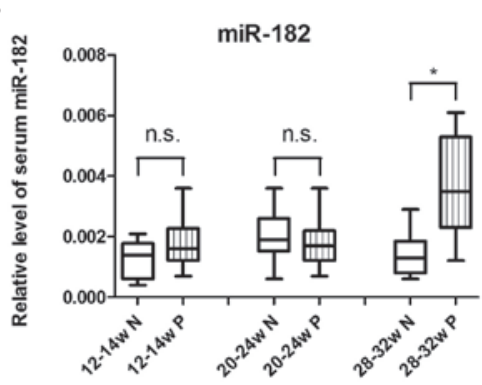

D

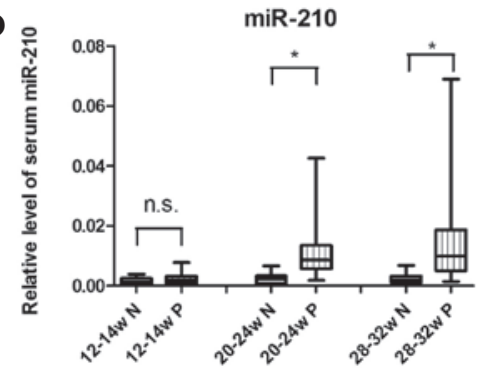

Figure 1. Box plot of the relative levels of serum (A) miR-152, (B) miR-182, (C) miR-183 and (D) miR-210 in different pregnancy trimesters. *P<0.01; n.s., no significance; N, normal; P preeclampsia.

A

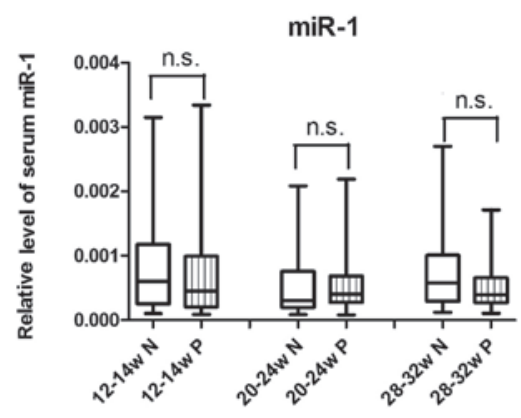

C

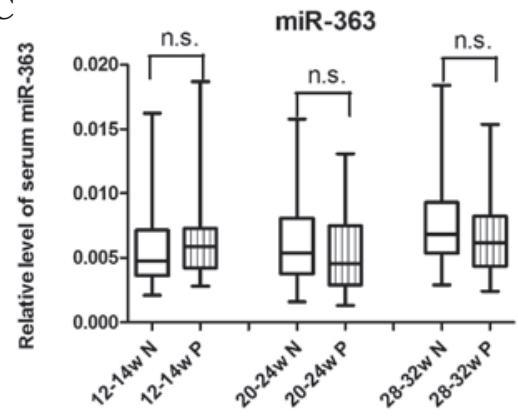

$\mathbf{E}$

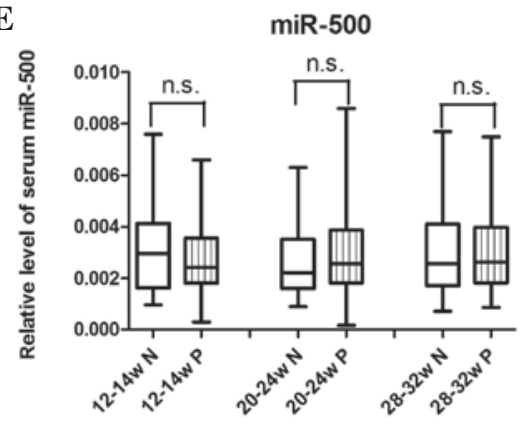

B

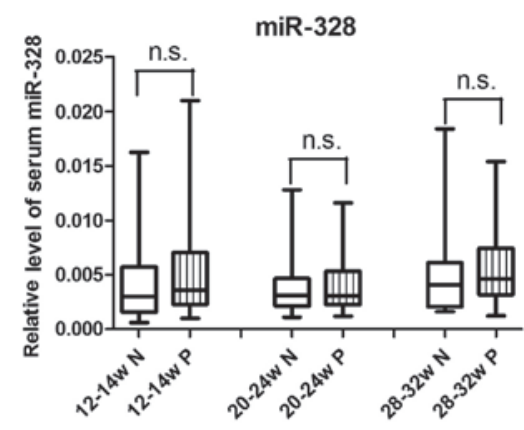

D

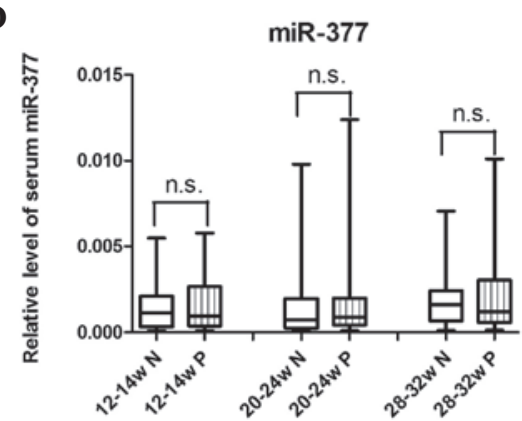

$\mathbf{F}$

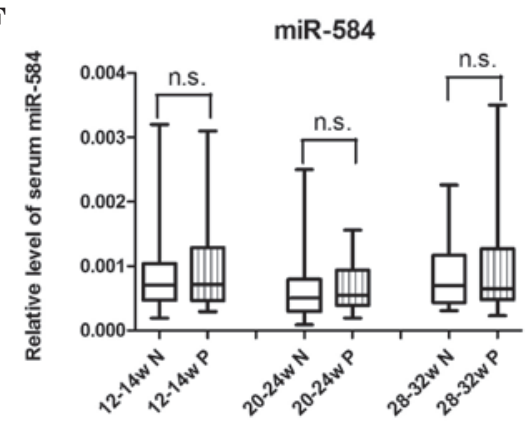

Figure 2. Box plot of the relative level of serum (A) miR-1, (B) miR-328, (C) miR-363, (D) miR-377, (E) miR-500 and (F) miR-584 in different pregnancy trimesters. n.s., no significance; $\mathrm{N}$, normal; $\mathrm{P}$, preeclampsia. 
A

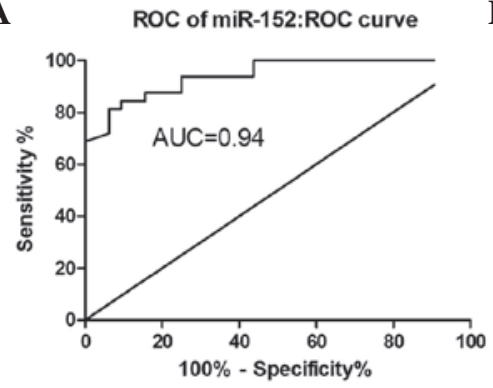

B

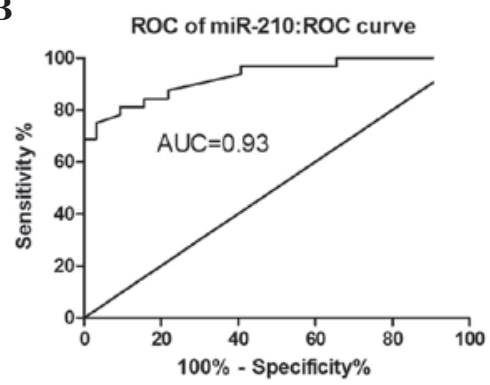

C

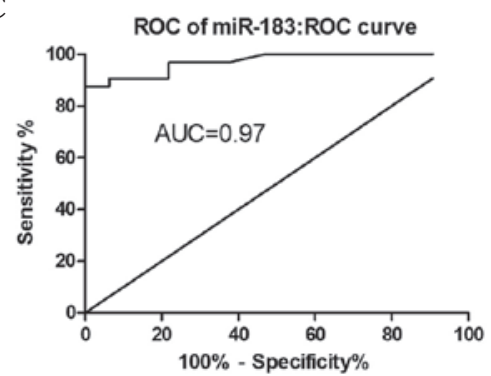

Figure 3. Receiver operating characteristic (ROC) curves of (A) miR-152, (B) miR-183 and (C) miR-210 during the 20-24th gestational weeks. AUC, area under curve.

\section{Discussion}

Circulating miRNAs have emerged as novel potential biomarkers for a host of diseases due to their presence and stability in the circulation (8). Previous studies on placental miRNAs in PE have indicated the involvement of miRNAs in the pathophysiology of PE. Although several reports (11-15) have already revealed the probable usefulness of measuring certain miRs for predicting the occurrence of PE, there were few overlaps among those studies and more investigation is required in order to clarify the exact role of PE-related miRNAs. The present study has quantified the relative expression levels of 10 miRNAs in maternal serum that had been reported to be dysregulated in the PE placenta.

A total of 4 miRNAs (miR-152, miR-182, miR-183 and miR-210) were identified to be upregulated significantly in the third trimester PE sera. Notably, there were 3 miRNAs (miR-152, miR-183 and miR-210) that showed significant elevation in PE sera since the second trimester. Consistent with the present results, by analyzing 15 pregnancies with mild $\mathrm{PE}$ and 15 pregnancies with severe PE, Zhang et al (15) found significantly elevated expression of miR-210 in PE plasma and a correlation of high plasma miR-210 with PE severity. Another study conducted by Xu et al (16) found higher circulatory miR-210 in severe PE at gestational weeks 15-18 and at term than those in the normal controls. In the present study, due to the scarcity of severe PE (only 2 cases), the correlation of the expression level of miRNAs with PE severity were not analyzed. miR-210 was validated to be regulated by NF- $\mathrm{KB}$ transcriptional factor p50 and HIF-1 $\alpha$ under hypoxia (15). By targeting on hydroxysteroid dehydrogenase 1, Ephrin-A3 and Homeobox-A9, miR-210 was postulated to inhibit cell migration and vascular remodeling and thus was involved in defective placentation (17). For miR-152, inconsistent with the present results, Gunel et al (12) reported downregulated levels in PE plasma. However, no detailed data or figures concerning miR-152 expression were reported. The predicted target for miR-152 is HLA-G (18), which is downregulated in PE pregnancies (19), and to a certain extent supported the present results. As for miR-182 and miR-183, to the best of our knowledge, this is the first report that found their elevation in the serum in preeclampsic patients despite their dysregulated expression in PE placenta $(3,4,9)$. miR-182 is a putative regulator of the Bcl-2-like gene that controls apoptosis, and a possible angiogenesis regulator via angiogenin and VEGF-B (9). Apoptosis and angiogenesis are major mechanisms involved in the pathogenesis of PE $(20,21)$. Functional analysis indicated that miR-183 was involved in cell differentiation, apoptosis and cell invasion in various types of cancer (22). The study by Shi et al (23) revealed a promotional role of miR-183 in endometrial stromal cells and inhibition of miR-183 on cell invasive ability.

The other 6 miRNAs (miR-1, miR-328, miR-363, miR-377, miR-500 and miR-584) that were reduced in PE placenta showed no significant differences in PE sera throughout the 3 trimesters. This phenomenon may be contributed to excessive placental trophoblast apoptosis accompanied in $\mathrm{PE}$, which offset the reduction of miRNAs in the placenta. Subsequently, generation sequencing in 4 pregnancies with PE in the study by Yang et al (14) identified 22 differential miRs during the third trimester of gestational age. Among these 22 dysregulated miRs, only 3 miRs (let-7d, let-7f and miR-223) were previously reported as downregulated in placenta, suggesting a poor correlation of circulating miRs in serum with miRs in placenta. Another study by Wu et al (24), using microarray analysis, identified 13 upregulated and 2 downregulated microRNAs in severe preeclamptic plasma during the 37-40th gestation weeks. Further RT-qPCR validated 7 elevated circulating microRNAs (miR-24, miR26a, miR-103, miR-130b, miR-181a, miR-342-3p and miR-574-5p). Hromadmikova et al (11) reported upregulation of circulating C19MC microRNAs (miR-516-5p, miR-517*, miR-520a*, miR-525 and miR-526a) in an established PE patient. The present results have few overlaps with these studies and no agreement was found among these researches. The inconsistency may be due to different gestational age, disease severity and experimental methods.

In the present study, blood was only sampled from pregnancies at 3 fixed gestational week periods instead of at the time of PE diagnosis. Among the $32 \mathrm{PE}$ cases, only 2 cases were diagnosed as PE before the 32nd gestation week, the additional 30 cases were diagnosed as PE after the 33rd gestation week. A limitation of the study is that the diagnostic profiles of miR-152, miR-183 and miR-210 in PE were not analyzed.

In conclusion, the present study identified elevation of circulatory miR-152, miR-183 and miR-210 since the second trimester in the pregnancies complicated with $\mathrm{PE}$, which indicated the potential of extracellular miRNAs to discriminate the patients at risk of later development of PE from those with normal progressing pregnancies 8-10 weeks ahead of clinical manifestations and diagnostic signs. Considering the small cohort of patients, the conclusion requires further confirmation by a larger sample size and more thorough exploration prior to clinical practice. 


\section{Acknowledgements}

The present study program was supported by grants from the Shanghai Baoshan Science Foundation (grant no. 11-E-15) and the Shanghai Medical Health Development Foundation in China. The authors would like to thank Ms. Jin Minmin and Mr. Yan Bing for the serum sample collection and storage.

\section{References}

1. Duley L: The global impact of pre-eclampsia and eclampsia. Semin Perinatol 33: 130-137, 2009.

2. Redman CW and Sargent IL: Preeclampsia and the systemic inflammatory response. Semin Nephrol 24: 565-570, 2004.

3. Pineles BL, Romero R, Montenegro D, Tarca AL, Han YM, Kim YM, Draghici S, Espinoza J, Kusanovic JP, Mittal P, et al: Distinct subsets of microRNAs are expressed differentially in the human placentas of patients with preeclampsia. Am J Obstet Gynecol 196: 261.e1-6, 2007.

4. Zhu XM, Han T, Sargent IL, Yin GW and Yao YQ: Differential expression profile of microRNAs in human placentas from preeclamptic pregnancies vs normal pregnancies. Am J Obstet Gynecol 200: 661.e1-7, 2009.

5. Hu Y, Li P, Hao S, Liu L, Zhao J and Hou Y: Differential expression of microRNAs in the placentae of Chinese patients with severe pre-eclampsia. Clin Chem Lab Med 47: 923-929, 2009.

6. Enquobahrie DA, Abetew DF, Sorensen TK, Willoughby D, Chidambaram K and Williams MA: Placental microRNA expression in pregnancies complicated by preeclampsia. Am J Obstet Gynecol 204: 178. e12-21, 2011.

7. Ji L, Brkić J, Liu M, Fu G, Peng C and Wang YL: Placental trophoblast cell differentiation: Physiological regulation and pathological relevance to preeclampsia. Mol Aspects Med 34: 981-1023, 2013.

8. Mitchell PS, Parkin RK, Kroh EM, Fritz BR, Wyman SK, Pogosova-Agadjanyan EL, Peterson A, Noteboom J, O'Briant KC, Allen A, et al: Circulating microRNAs as stable blood-based markers for cancer detection. Proc Natl Acad Sci USA 105: 10513-10518, 2008.

9. Noack F, Ribbat-Idel J, Thorns C, Chiriac A, Axt-Fliedner R, Diedrich $\mathrm{K}$ and Feller AC: miRNA expression profiling in formalin-fixed and paraffin-embedded placental tissue samples from pregnancies with severe preeclampsia. J Perinat Med 39: 267-271, 2011.

10. Betoni JS, Derr K, Pahl MC, Rogers L, Muller CL, Packard E, Carey DJ, Kuivaniemi H, Tromp G: MicroRNA analysis in placentas from patients with preeclampsia: Comparison of new and published results. Hypertens Pregnancy 32: 321-339, 2013.
11. Hromadnikova I, Kotlabova K, Ondrackova M, Kestlerova A, Novotna V, Hympanova L, Doucha J and Krofta L: Circulating C19MC microRNAs in preeclampsia, gestational hypertension, and fetal growth restriction. Mediators Inflamm 2013: 186041, 2013.

12. Gunel T, Zeybek YG, Akçakaya P, Kalelioğlu I, Benian A, Ermis H and Aydınlı K: Serum microRNA expression in pregnancies with preeclampsia. Genet Mol Res 10: 4034-4040, 2011.

13. Hromadnikova I, Kotlabova K, Doucha J, Dlouha K and Krofta L: Absolute and relative quantification of placenta-specific micrornas in maternal circulation with placental insufficiency-related complications. J Mol Diagn 14: 160-167, 2012.

14. Yang Q, Lu J, Wang S, Li H, Ge Q and Lu Z: Application of next-generation sequencing technology to profile the circulating microRNAs in the serum of preeclampsia versus normal pregnant women. Clin Chim Acta 412: 2167-2173, 2011.

15. Zhang Y, Fei M, Xue G, Zhou Q, Jia Y, Li L, Xin H and Sun S: Elevated levels of hypoxia-inducible microRNA-210 in pre-eclampsia: New insights into molecular mechanisms for the disease. J Cell Mol Med 16: 249-259, 2012.

16. Xu P, Zhao Y, Liu M, Wang Y, Wang H, Li YX, Zhu X, Yao Y, Wang H, Qiao J, et al: Variations of microRNAs in human placentas and plasma from preeclamptic pregnancy. Hypertension 63: 1276-1284, 2014.

17. Ishibashi O, Ohkuchi A, Ali MM, Kurashina R, Luo SS, Ishikawa T, Takizawa T, Hirashima C, Takahashi K, Migita M, et al: Hydroxysteroid (17- $\beta$ ) dehydrogenase 1 is dysregulated by miR-210 and miR-518c that are aberrantly expressed in preeclamptic placentas: A novel marker for predicting preeclampsia. Hypertension 59: 265-273, 2012.

18. Tan Z, Randall G, Fan J, Camoretti-Mercado B Brockman-Schneider R, Pan L, Solway J, Gern JE, Lemanske RF, Nicolae D, et al: Allele-specific targeting of microRNAs to HLA-G and risk of asthma. Am J Hum Genet 81: 829-834, 2007.

19. Yie SM, Li LH, Li YM and Librach C: HLA-G protein concentrations in maternal serum and placental tissue are decreased in preeclampsia. Am J Obstet Gynecol 191: 525-529, 2004.

20. Heazell AE and Crocker IP: Live and let die-regulation of villous trophoblast apoptosis in normal and abnormal pregnancies. Placenta 29: 772-783, 2008.

21. Smith GC and Wear H: The perinatal implications of angiogenic factors. Curr Opin Obstet Gynecol 21: 111-116, 2009.

22. Zhang QH, Sun HM, Zheng RZ, Li YC, Zhang Q, Cheng P, Tang ZH and Huang F: Meta-analysis of microRNA-183 family expression in human cancer studies comparing cancer tissues with noncancerous tissues. Gene 527: 26-32, 2013.

23. Shi XY, Gu L, Chen J, Guo XR and Shi YL: Downregulation of miR-183 inhibits apoptosis and enhances the invasive potential of endometrial stromal cells in endometriosis. Int J Mol Med 33: 59-67, 2014.

24. Wu L, Zhou H, Lin H, Qi J, Zhu C, Gao Z and Wang H: Circulating microRNAs are elevated in plasma from severe preeclamptic pregnancies. Reproduction 143: 389-397, 2012. 